\title{
Aprendizaje activo a nivel post-secundario para promover el desarrollo de gestión de competencias empresariales sobre liderazgo en respuesta a los requerimientos del mercado laboral de Puerto Rico
}

\author{
Ángel L. Rivera Aponte / alra71@yahoo.com \\ Universidad de Puerto Rico \\ Recinto de Río Piedras \\ Carmen I.Figueroa Medina / cfigueroafae@gmail.com \\ Universidad de Puerto Rico \\ Recinto de Mayagüez
}

\section{RESUMEN:}

El aprendizaje activo a nivel universitario es imprescindible para promover el desarrollo de competencias empresariales ante los requerimientos actuales del mercado laboral en Puerto Rico. La participación del estudiantado matriculado en el curso de Liderazgo en Administración de Empresas, utilizando el proceso de inducción generado a través de la integración de teoría y práctica, promovió el desarrollo de las referidas competencias. Un análisis cualitativo demostró la efectividad del proceso de enseñanza y aprendizaje mediante metodologías pedagógicas de aprendizajeactivo. Los resultados se evidenciaron a través del aprovechamiento del estudiantado en el proceso de enseñanza y aprendizaje aumentando su nivel de competencias de liderazgo, entre otras.

Palabras clave: aprendizaje activo, liderazgo, proceso enseñanza y aprendizaje y desarrollo en la gestión de talento humano

\section{ABSTRACT:}

Active learning at undergraduate level is necessary to promote business competencies on behalf of the actual labor market in Puerto Rico. The participation of students registered in the Leadership course at the School of Business, using the induction process, generates throughout the integration of theory and practice the encouragement of competencies development. Qualitative analysis demostrates the effectiveness of the teaching and learning experience through pedagogical methodologies of active learning. Results obtained by students' success during the teaching and learning experience increased the competencies level of leadership, etc.

Keywords: active learning, leadership, teaching and learning experience, development of human capital 


\section{INTRODUCCIÓN}

En la sociedad del conocimiento en que nos encontramos, las instituciones de educación superior tienen un rol crucial en la generación y transmisión del conocimiento. Dado a las continuas exigencias del mercado laboral, la educación post-secundaria deberá integrarse más desde la perspectiva de planificación de recursos humanos y los procesos de contribuir, promover y gestar una fuerza laboral capacitada. Debemos señalar que los requerimientos del mercado laboral son cada más vez rigurosos y buscan que sus candidatos posean competencias cognoscitivas, conductuales y afectivas desde una perspectiva holística e integradora. La sociedad del conocimiento requiere también que todos sus miembros aprendan a aprender, ya que la información y el conocimiento son las herramientas más valiosas en cualquier mercado que se considere competitivo. Desde el campo de la Administración de Empresas, uno de los recursos fundamentales se centra en el capital humano. El capital humano, o sea, la integración de la suma de los conocimientos, las destrezas, habilidades y actitudes y la experiencia que poseen todos los miembros de una empresa, constituye el recurso más valioso para la producción y la creación organizacional. De esta forma, el desarrollo de las competencias del capital humano a través de las instituciones post-secundarias puede contribuir como una de las ventajas competitivas añadidas de una empresa. Un informe del Departamento del Trabajo de Puerto Rico (2005) sobre un estudio de destrezas y ocupaciones de mayor demanda indicó que las competencias requeridas por el mercado laboral de la Isla son las siguientes:

capacidad de comunicación oral y escrita, desarrollo de destrezas analíticas, uso de herramientas de tecnología de la información, desarrollo de destrezas de liderazgo, trabajo en equipo, relaciones interpersonales, desarrollo de valores éticos y sociales, capacidad de razonamiento crítico y reflexivo, capacidad de responsabilidad social y comunitaria, desarrollo de la destreza de toma de decisiones y solución de problemas, desarrollo del aprendizaje continuo y desarrollo del conocimiento, entre otras. (p. 53) 
Ésta es una de las razones por la cual en las últimas décadas, las instituciones de educación superior en la Isla se encuentran inmersas en procesos de creación de programas nuevos y modificaciones curriculares, entre otras actividades académicas, con el objetivo de cumplir con las exigencias internas y externas de su entorno. Por otra parte, las instituciones académicas buscan atemperar sus currículos a las exigencias del mercado laboral promoviendo la gestión del talento humano. De esta forma, la Facultad de Administración de Empresas (FAE) del Recinto de Río Piedras de la Universidad de Puerto Rico (UPR-RRP), se encuentra en el proceso de implementación, evaluación concienciación y divulgación de un nuevo currículo. A partir de la implementación del nuevo currículo promueve el desarrollo de las siguientes competencias en el currículo de modo trasversal con el objetivo de que el estudiantado adquiera los saberes a través de su educación. Según un informe de la UNESCO (2003), la educación es el principal agente de la transición hacia el desarrollo sostenible, incrementando la capacidad de las personas de hacer realidad sus concepciones de la sociedad. La educación no se limita a impartir capacidades científicas y técnicas, también refuerza la motivación, la justificación y el apoyo social a las personas que los buscan y los aplican. Cabe señalar que la mayor parte de la formación de capital humano es el resultado de una enseñanza formal.

La educación ha constituido siempre un elemento fundamental en el desarrollo de los pueblos civilizados (Claudio Tirado, 2002). Ésta provee la base y, en una buena medida, determina el progreso que el pueblo puede alcanzar en sus diferentes manifestaciones del ambiente externo. Como institución, creada por la sociedad, la universidad tiene la responsabilidad de impartir enseñanza de la más alta calidad a la ciudadanía del pueblo.

Desde esta perspectiva, este estudio examina cualitativamente la efectividad de la experiencia pedagógica en la implementación de un nuevo currículo a través del proceso de enseñanza y aprendizaje del curso de Liderazgo ${ }^{1}$. En el mismo se utilizó la modalidad de

${ }^{1}$ El curso de Liderazgo está codificado con las siguientes siglas ADMI 4415. Cabe señalar que es la primera vez que el curso se ofrece en la Facultad de Administración de Empresas del Recinto de Río Piedras en la Universidad de Puerto Rico. 
aprendizaje activo y cooperativo que facilita el desarrollo de competencias en el estudiantado antes los requerimientos del mercado laboral puertorriqueño. De esta forma, esta investigación aporta información, conclusiones y recomendaciones en el proceso de implementación del aprendizaje activo y cooperativo en el referido curso.

En este análisis, pretendemos considerar elementos referentes a las estrategias de enseñanza y aprendizaje utilizando la modalidad de aprendizaje activo y cooperativo en la implantación del curso de Liderazgo. Es importante señalar que en la FAE, en la UPR-RRP, esta modalidad de enseñanza es innovadora siendo que sus resultados han sido exitosos tanto para el cuerpo docente, como para el estudiantado. De igual forma, el proceso de avalúo ${ }^{2}$ se llevó a cabo para medir el aprovechamiento académico del estudiantado. Además, los estudiantes realizaron su propio avalúo sobre la experiencia del curso a través de un "video assessment" ${ }^{3}$. Los resultados del proceso de la misma forma han promovido un interés académico en otros facultativos dentro del Recinto de Río Piedras. El desarrollo de liderazgo como el compromiso de la Facultad hacia el desarrollo de la gestión del talento humano en el estudiantado antes las exigencias del mercado laboral actual, han sido esbozadas dentro de la FAE desde el enunciado de su misión: "Desarrollar líderes gerenciales, empresariales y académicos, profesionales y éticos mediante una educación de excelencia e iniciativas de investigación y servicio en el contexto de Puerto Rico y el mundo”. Asimismo, el compromiso de la Facultad hacia el desarrollo de la gestión del talento humano en el estudiantado antes las exigencias del mercado laboral actual, ha sido esbozada dentro del "Perfil de Egresado". Las ochos áreas

\footnotetext{
${ }^{2}$ Según Verdejo y Medina (2008), el avalúo se refiere a recoger, organizar y sintetizar información diversa para distintos fines, entre los cuales está facilitar la evaluación del aprendizaje y tomar decisiones relacionadas. Esta información puede ser de índole cualitativa o cuantitativa.

${ }^{3}$ El estudiantado utilizó un "video-assessment" como una herramienta para llevar a cabo un proceso de reflexión y auto-avalúo referente a la experiencia sobre el aprendizaje adquirido a través del curso. El estudiantado avalúo el contenido, metodologías de aprendizaje y cumplimiento de los requisitos mínimos del curso de liderazgo según el prontuario.
} 
relevantes de este perfil implican el análisis crítico en la toma de decisiones, aplicación de las destrezas cuantitativas y cualitativas del análisis gerencial y empresarial, conocimiento gerencial en el ámbito local e internacional, aplicación de principios y valores éticos, comunicación efectiva, capacidad para trabajar en equipo, establecer relaciones interpersonales, sensibles a la diversidad cultural y humana, y el uso de la competencias tecnológicas, de información e investigación para su crecimiento profesional continuo. Las áreas relevantes han tomado sentido al facilitar la gestión del talento humano, utilizando las experiencias de aprendizaje activo y cooperativo en los estudiantes en el curso de liderazgo. Este estudio contribuye al entendimiento de un modelo educativo para la formación académica en el campo de la Administración de Empresas por medio del desarrollo de gestión del talento humano a través de la competencia de liderazgo.

\section{REVISIÓn DE LITERATURA}

El aprendizaje activo comenzó a tomar relevancia en las disciplinas de las ciencias naturales, desde la década pasada. La atención de la academia ha aumentado el valor del aprendizaje activo en el salón de clase en el ámbito universitario (Caccavo, 2001; Lunsford \& Herzog, 1997; Tessier, 2004, 2006, 2007). Varios estudios indican que el aprendizaje activo mejora el aprendizaje estudiantil y su desempeño (Crouch \& Mazur, 2001; Knight \& Wood, 2005; Tesier, 2007; Mazur, 1997). Por tal razón, un método tradicional, tal como dictar una clase, no es un método a través del cual se capte una mayor atención del estudiantado. Una clase por sí sola no se considera la forma más efectiva para promover el aprendizaje (Handelsman, et al., 2004; Knight \& Wood, 2005).

Según Silberman (1998), los aprendices adultos poseen una diversidad de estilos utilizando entre otros: la habilidad de escuchar, observar, hablar y accionar. Ello implica que el proceso de aprendizaje estudiantil se ha tornado un proceso más natural. Meier (2000) define aprendizaje activo cómo el modo en que las personas aprenden naturalmente. Sin embargo, Petress (2008) señala que el aprendiz activo no depende totalmente del docente, sino que el aprendizaje 
activo convierte al estudiante en un socio del proceso de aprendizaje. El aprendizaje activo ha sido enlazado al pensamiento crítico y a los aumentos en los niveles de interacción social, resultando en un compromiso institucional consecutivo, y en el enaltecimiento del bienestar y crecimiento personal de los estudiantes (Braxon, Milem \& Sullivan, 2000).

Todo proceso de aprendizaje requiere que el estudiante construya el significado del aprendizaje activamente. Los estudiantes fundamentan este significado tomando la nueva información y relacionándola con el conocimiento propio. Posteriormente, usará estos nuevos conocimientos para razonar y solucionar problemas. En este proceso cada persona está continuamente cotejando esta nueva información contra las viejas y cuando surgen discrepancias se llega a nuevos conocimientos y construcciones de la realidad (Brooks, 1989). Por ello, una de las tareas fundamentales del docente es explorar lo que los estudiantes traen, para ayudarlos a orientar y redefinir el entendimiento de esos conceptos.

El método de aprendizaje activo en el salón de clase pretende que los estudiantes reflexionen sobre la manera de escuchar y accionar sobre los conceptos teóricos. Asimismo, el aprendizaje activo permite que los estudiantes tornen su posición de espectadores hacia un mayor compromiso en las actividades de proceso de aprendizaje y el desarrollo de habilidades que le permitan asimilar más hábilmente los conceptos teóricos. De esta forma, el estudiante experimenta un incremento en la motivación y desarrolla el compromiso continuo hacia el desarrollo de una educación superior.

\section{Metodología}

Este estudio trata sobre el aprendizaje activo y cooperativo en un grupo de 37 estudiantes matriculados en el curso de Liderazgo (ADMI-4415) en la FAE en la UPR-RRP. La metodología de estudio es de carácter activo, experiencial y participativo que desarrolla en los estudiantes universitarios las estrategias cognoscitivas, conductuales y afectivas (Keyser, 2000; McKeachie, 1999; Silberman, 1996; Meyers \& Jones, 1993; Bonwell \& Eison, 1991). Del mismo modo, desarrolla las habilidades cooperativas mejorando su aprovechamiento acadé- 
mico, promoviendo el enriquecimiento tanto personal como social. Se diseña una intervención educativa a nivel universitario a través de la dinámica de grupo, concretizada en una serie de objetivos propuestos a través del curso de Liderazgo. Por un lado, se desarrollan e integran aportaciones teóricas para una mejor comprensión del trabajo en equipo y del aprendizaje activo y cooperativo. Entre las técnicas de aprendizaje utilizadas podemos mencionar las siguientes: tormenta de idea, diario reflexivo, discusión de películas, prueba de comprensión del material, trabajo en grupos cooperativos, debates, compartir tareas, ejercicios de aplicación de los conceptos teóricos en situaciones de la vida real o situaciones nuevas e inesperadas y mapas conceptuales, entre otros. Durante el proceso se llevaron a cabo actividades para promover el crecimiento personal del estudiantado, un proceso de retroalimentación a través del diálogo, la reflexión y el pensamiento crítico de los estudiantes.

En la implementación de la experiencia pedagógica del curso de Liderazgo diseñamos métricas para evaluar el aprovechamiento académico del estudiantado. Desde este contexto, el estudiando tomó la iniciativa de autoevaluar la experiencia pedagógica adquirida durante el trayecto el curso. De igual forma, se desarrolló una actividad de grupo focal como proceso de reflexión, modificación y recomendaciones de la experiencia relacionada al aprendizaje en la competencia de liderazgo ${ }^{4}$. Por otra parte, los estudiantes desarrollaron un instrumento para avaluar el proceso de enseñanza y aprendizaje a través de un video. En el mismo se recogen su experiencia en cuanto a contenido, diseño e integración de competencias, entre otros.

\section{Discusión}

En la Conferencia Mundial sobre la Educación (1998), se señala que existe una demanda de educación superior sin precedentes, acompañada de una gran diversificación de la misma y una mayor

\footnotetext{
${ }^{4}$ Quienes investigan el liderazgo están más en desacuerdo de lo que se puede imaginar en cuanto a lo que el liderazgo es. Este desacuerdo se deriva de que el liderazgo es un fenómeno complejo que involucra al líder, a los seguidores y la situación (Hughes, Ginnett \& Curphy, 2007).
} 
toma de conciencia de la importancia fundamental que este tipo de educación reviste para el desarrollo sociocultural y económico y para la construcción del futuro de cara a al cual las nuevas generaciones deberán estar preparadas con nuevas competencias y nuevos conocimientos e ideales. Estas competencias deberán estar integradas a las exigencias del mercado laboral local e internacional. Las mismas se pueden adquirir a través de proceso de formación integral basada en el conocimiento, el desarrollo en la gestión del talento humano y la experiencia como resultado voluntario del individuo. La importancia del aprendizaje para los profesionales está ampliamente reconocida; implícitamente, es el recurso principal que contribuye alcanzar la ventaja competitiva de una corporación (Argyris, 1991; Senge, 1990).

En los últimos años, en vías de alcanzar tanto la reflexión académica como en un ambiente de aprendizaje organizacional, los aprendices necesitan compartir las reflexiones y experiencias, el apoyo y coraje, diseminar las ideas y sentimientos hacia afuera, y asimilar los puntos de vistas de otros (Hodgkinson \& Brown, 2003). Desde esta perspectiva, el estudiantado matriculado en el curso de liderazgo adquiere el aprendizaje a través del proceso de reflexión guiado por la estrategia del docente. "Una estrategia de enseñanza es un plan general de actividades o interacciones entre profesor y estudiantes dirigido a suscitar el aprendizaje". "Consiste de una secuencia de actividades o secuencia de interacciones y comunicaciones entre el profesor y el estudiante que crean las condiciones que promueven el aprendizaje" (Villarini, 1991, p. 28).

\section{EXPERIENCIA PEDAGÓGICA EN EL CURSO DE LIDERAZGO}

A los 37 estudiantes del curso durante la primera semana de clase se les suministró un ejercicio académico con el objetivo de auscultar qué conocimiento tenían sobre liderazgo, qué esperaban del curso, cuál fue su motivación para matricularse en el curso, cuáles áreas necesitaban mejorar, cuál año de estudio cursaban, entre otros. Debemos señalar que este ejercicio se realizó para determinar cuál era el perfil del estudiantado, sus intereses y necesidades. Varios de los hallazgos fueron los siguientes: el grupo estaba compuesto de estudiantes que apenas comenzaban su primer año de estudio, hasta 
estudiantes que habían obtenido su grado de Bachiller en Administración de Empresas. Se encontraron limitaciones en la gran mayoría de los estudiantes del curso. Estos manifestaron que tenían miedo de expresar su forma de pensar, actuar, compartir, participar en clase, no deseaban trabajar en equipo, ni compartir entre ellos, entre otras. Uno de los grandes retos del curso de liderazgo fue centrado en la planificación y diseño de los conceptos teóricos alineados a la metodología del aprendizaje activo y cooperativo, debido a la diversidad de los estudiantes (nueve estudiantes con condiciones especiales ${ }^{5}$ ). Por otra parte, los estudiantes indicaron que su interés en el curso consistía en la adquisición de conocimiento o algunas técnicas.

Los estudiantes pasaron por un proceso de inducción a través del método de enseñanza de aprendizaje activo y cooperativo. Se realizaban actividades una vez a la semana con el objetivo que se integrara la teoría y la práctica. De este modo, se generaba en ellos un proceso de socializar, integrar y compartir el conocimiento. De esta forma, se continuaba generando las estrategias de aprendizaje activo y cooperativo que incluía una variedad de estrategias tales como conferencias activas, utilización de películas, ejercicios de aplicación de un escenario real y situaciones imprevistas o nuevas. Estas estrategias antes mencionadas se comenzaron a llevar a cabo por la diversidad del grupo. Debido a la composición heterogénea del grupo, el estudiantado en ocasiones no respetaba la diversidad, la opinión de sus compañeros y expresaba verbalmente su resistencia al cambio.

Durante el semestre, los estudiantes de liderazgo realizaron ejercicios individuales (respuesta afectiva, diario reflexivo, prueba de comprensión del material, pausa de aclaración, tormenta de ideas, entre otros). El objetivo de los ejercicios individuales se realizaba

${ }^{5}$ Ley 51 de 1996. Ley de Servicios Educativos Integrales para personas con impedimentos. La UPR en RRP considera como condiciones especiales las siguientes: condición visual, condición auditiva, condición del habla, condición emocional, enfermedad cardiovascular, perlesía cerebral, epilepsia, distrofia muscular, condición renal, condición respiratoria, condición ortopédica, enfermedad sexualmente transmisible, diabetes, paraplejia, artritis y problemas de aprendizaje. 
por dos criterios principales: (1) como proceso de evaluación formativa (exámenes) y (2) como proceso retroalimentación de los temas asignados y discutidos en el curso. En estas actividades los estudiantes comenzaron a interactuar, socializar y reflexionar sobre aspectos éticos, respeto a la diversidad, y su rol dentro y fuera de su responsabilidad social. Los estudiantes comenzaron a adoptar un lema: "convivir es vivir".

Cada vez que se impartía el curso se comenzaba a incorporar otras estrategias de enseñanza y aprendizaje por vía del proceso en la experiencia pedagógica activo y cooperativo. Las estrategias se iban implementando una vez el estudiantado entendía y comprendía la noción teórica. Debemos señalar, que el liderazgo no se desarrolla solamente desde el contexto teórico; para promover la competencia se requiere de ejercicios integración práctica. De este modo, se comenzó con la metodología de aprendizaje cooperativo (grupos cooperativos, sesiones de repaso activo, trabajo en pizarra, mapas conceptuales, juego de roles, discusión en panel y debates). De aquí en adelante, el proceso de participación del estudiantado cambió substancialmente. Ejemplos del cambio se denotan en los debates sobre algunos pensamientos tales como: "el liderazgo es tanto una ciencia como un arte", "el liderazgo es tanto racional como emocional”, "el líder nace o se hace”, entre otros. Durante las discusiones en el aula, los estudiantes participaron en los juegos de roles y comenzaron a desarrollar técnicas de estudios grupales realizando como guía de estudios elaboraciones de mapas conceptuales. También debemos mencionar que se comenzaron a generar equipos de trabajos cooperativos. Los trabajos en equipos contribuyeron en aumentar su nivel de aprovechamiento, relaciones interpersonales, comunicación, negociación, manejo de conflicto y discusiones dentro y fuera del salón de clase, sobre los temas y ejercicios realizados.

Una actividad llevada a cabo a través de aprendizaje cooperativo en el curso de liderazgo consistió en la realización de una entrevista a un líder. Los estudiantes llevaron a cabo una tormenta de ideas para identificar a quién entrevistarían. Además, la asignación de ejercicios en equipo, promovió el desarrollo de las destrezas mentales de carácter individual y grupal. Entre los requisitos del curso, 
los estudiantes tenían que presentar informes orales y escritos para determinar cómo establecieron la definición del problema, cómo generaban las alternativas para solucionar un problema, entre otros. El nivel de integración de los estudiantes continuó aumentando, al igual que su nivel de participación, el respeto a la diversidad, la solidaridad, como también, el compromiso en cuanto a las actividades realizadas y asignadas en el curso.

Sin embargo, durante el proceso nos encontramos con una serie de barreras tales como: la diversidad del estudiantado, el nivel educativo por año académico, el espacio físico, la cantidad de estudiantes matriculados, la construcción en cuanto la planificación y el diseño del curso relacionado al proceso de enseñanza y aprendizaje, la disposición del estudiante al participar de esta modalidad de enseñanza, la innovación de nuevas alternativas de enseñanza no tradicional en el Recinto de Río Piedras de la Universidad de Puerto Rico, entre otras. Otras de las situaciones consideradas relevantes son, las diferencias en actitudes, valores y creencias de cada generación en su impacto en la forma en cómo consideran el liderazgo y la propuesta de los estilos por generación.

\section{Diálogo del Grupo Focal con el estudiantado}

De los resultados del grupo focal, se evidenció en el estudiantado un alto nivel de satisfacción en cuanto a la experiencia pedagógica en el curso sobre liderazgo. Mediante el uso del aprendizaje activo y cooperativo los estudiantes maximizaron la captación de los conceptos teóricos disminuyendo el nivel de incertidumbre con la terminología utilizada en el curso. Comentaron que la disponibilidad del docente para aclarar las dudas durante el proceso de reflexión y las dinámicas aumentó la espontaneidad del estudiantado a exponer cuestionamientos acerca de los temas sobre liderazgo. También, expresaron que la funcionalidad del aprendizaje activo y cooperativo mediante dinámicas y ejercicios los ayudó a lidiar con el grado de dificultad en la comprensión de los conceptos.

De la misma forma, el estudiantado manifestó que las estrategias pedagógicas sobrepasaron sus expectativas. El proceso de enseñanza y aprendizaje contribuyó en aumentar su capacidad en entender el concepto de liderazgo. Durante el proceso académico del curso de 
Liderazgo, reconocieron que se realizaron una variedad de actividades, dinámicas, ejercicios y exámenes que apoyaron su aprendizaje de forma tal que impactó tanto su vida estudiantil como personal. Los estudiantes expresaron que durante el curso de Liderazgo comenzaron a notar cambios en su comportamiento mediante un proceso reflexivo (por ejemplo: vestimenta, comunicación verbal y escrita, y el desarrollo de un pensamiento crítico, entre otros). El estudiantado manifestó que el desarrollo de las competencias relacionadas a la gestión de talento humano mediante el uso del aprendizaje activo y cooperativo, contribuirá a su desarrollo profesional.

Por otra parte, el estudiantado recomendó: una continuidad a la secuencia curricular en liderazgo, la creación de un internado, donde se le permitiera realizar trabajo de investigación con la comunidad interna y externa, y que el modelo de enseñanza y aprendizaje activo y cooperativo fuera imitado en otros cursos de la Facultad de Administración de Empresas, entre otros. Sin embargo, algunos estudiantes señalaron que el libro de texto deberá ser reconsiderado debido a la profundidad de su contenido. De la misma forma, puntualizaron que los profesores minimizaron la brecha del grado de dificultad del texto, mediante el uso de dinámicas y ejercicios.

\section{DISEÑO Y PLANIFICACIÓN POR PARTE DEL DOCENTE}

Para el desarrollo más eficaz del proceso educativo en un "nuevo escenario educativo" se hace imperativo que los diferentes funcionarios funjan como líderes efectivos (Claudio Tirado, 2002). Estos deberán exhibir los atributos como: visión, pasión, integridad, confianza, curiosidad, creatividad y osadía. Estos elementos han sido señalados en la literatura pedagógica reciente como esenciales en la función de líderes educativos transformadores (Anderson, 1996). Sullivan y Glanz (2000) afirman que un líder educativo que entiende la historia de la supervisión y que también entiende los cambios y demandas de la sociedad estará mejor capacitado para afrontar los retos que surgen diariamente en los escenarios de trabajo.

Uno de los retos del docente es saber seleccionar y desarrollar hábilmente las estrategias de aprendizaje idóneas para cada clase. Esta estrategia promoverá constructivamente la discusión entre el 
profesor y el estudiante. De esta manera, las estrategias utilizadas contribuirán en incrementar la retención del contenido, mejorando así la habilidad de maximizar el nivel cognoscitivo, conductual, el interés y la motivación. El uso de la metodología de aprendizaje activo y cooperativo en el salón de clase permite que el estudiante asuma la responsabilidad de su aprendizaje. Mientras que el docente deberá continuar planificando las actividades de aprendizaje activo para cada clase según el tema de discusión. De este modo, se promueve la diversidad en las estrategias pedagógicas para romper con paradigmas tradicionales de enseñanzas.

El principio de la planificación es la formulación de los objetivos que se derivan del prontuario del curso. El segundo paso es la selección de las estrategias puntualizando en tres actividades de aprendizaje dónde se desarrolle la introducción del tema, la discusión de los conceptos teóricos, y termine con un ejercicio de reflexión. De igual forma, el aprendizaje activo promueve la proposición de actividades adicionales tales como asignación de lecturas, ejercicios, preparación de conferencias y proyectos.

La consideración previa de las características y el tamaño del grupo que va a experimentar las actividades de aprendizaje activo toma relevancia. Una de la limitaciones principales para la efectividad del proceso de enseñanza y aprendizaje es que a mayor la cantidad de estudiantes por sección académica mayor será la dificultad y mayor será el esfuerzo de parte del docente para lograr cumplir con los objetivos de aprendizaje propuesto en el curso.

Por otra parte, antes de comenzar las actividades de aprendizaje, el docente debe preparar a los estudiantes hacia la disponibilidad de aprender activamente. Es menester del docente ofrecer las instrucciones claras y explícitas respetando los estilos de aprendizaje de los estudiantes, y procesar las respuestas dentro de un clima de respeto y retroalimentación. De la misma forma, el docente, deberá generar un "empowerment" en el estudiantado, con el objetivo de que promuevan el rol de mentor entre sus pares. De esta forma, se genera en el estudiantado el compromiso en sí mismo en la búsqueda de nuevo conocimiento.

La etapa práctica del aprendizaje le permite al docente colaborar en la captación y maximización de los conceptos teóricos del tema 
expuesto durante la clase. Asimismo, se promueve la interacción entre el estudiantado hacia el reconocimiento de la diversidad de pensamiento de un concepto, de un modelo o una teoría. Según Meier (2000), la fase práctica ayuda a los aprendices en la integración e incorporación del conocimiento o destrezas nuevas en una diversidad de formas tales como simulaciones de la vida real, ejercicios de aprendizaje en acción, actividades de solución de problemas, reflexión y articulación individual, debates o diálogos grupales, actividades de construcción de destrezas, entre otros. Cabe señalar que una de las tareas fundamentales del docente es explorar lo que los estudiantes traen para ayudarlos a redefinir el entendimiento de esos conceptos. De la misma forma, el facultativo tiene que tomar en cuenta "el equipaje" de la andragogía.

\section{AUTO-AVALÚO POR PARTE DEL ESTUDIANTADO}

Los instrumentos de auto-avalúo diagnóstico y ejercicio reflexivos pueden ser particularmente de gran valor para los estudiantes en los cursos de gerencia y empresarismo (Human, Clark \& Baucus, 2005). Mediante el "video assessment" los estudiantes expresaron que se cumplió el contenido, evidenciaron que la modalidad de enseñanza y aprendizaje fue efectivo sobre pasando las expectativas teóricopráctico del periodo lectivo (enero-mayo, 2009). Los resultados han transcendido fuera de las esferas universitarias.

\section{CONCLUSIONES Y RECOMENDACIONES}

La educación superior se enfrenta en todas partes desafíos y dificultades relativos a la financiación, la igualdad de condiciones de acceso a los estudios y en el transcurso de los mismos, una mejor capacitación del personal, la formación basada en las competencias, la mejora y conservación de la calidad de la enseñanza, la investigación y los servicios, la pertinencia de los planes de estudios, las posibilidades de empleo de los diplomados, el establecimiento de acuerdos de cooperación eficaces y la igualdad de acceso a los beneficios que reporta la cooperación internacional (UNESCO, 1998). La educación superior debe hacer frente a la vez a los retos que suponen las nuevas oportunidades que abren las tecnologías, que mejoran la ma- 
nera de producir, organizar, difundir y controlar el saber y de acceder al mismo.

Estos retos se encuentran en el desarrollo de la gestión talento humano. La experiencia pedagógica en la implementación de un nuevo currículo en la Facultad de Administración de Empresas, específicamente en la enseñanza y aprendizaje del curso de liderazgo, fue una enriquecedora. Concordamos con Davis (1993) que la instrucción basada en experiencias de campo es una forma activa de aprendizaje que ha probado la maximización de los resultados del aprendizaje estudiantil, incluyendo los aspectos de retención y el mejoramiento de las destrezas de solución de problemas de los estudiantes. Por otra parte, al igual que Hickcox (2002), entendemos que las experiencias de campo son centradas en los aprendices, permitiéndoles a los estudiantes la oportunidad de aplicar las ideas y los conceptos dictados en un salón de clase tradicional hacia un ambiente que estimula el pensamiento crítico y el análisis.

Los estudiantes del curso de Liderazgo lograron maximizar varias áreas tales como su aprovechamiento académico, compromiso, trabajo en equipo, estrategias de manejo de conflicto, confianza en sí mismo, respeto a la diversidad, responsabilidad social, relaciones interpersonales, comunicación, valores, liderazgo, entre otros. Desde nuestra experiencia, el proceso de aprendizaje activo y cooperativo es una herramienta enriquecedora, que contribuye en añadir valor donde todos ganamos. De esta forma, señalamos que el proceso de enseñanza y aprendizaje en el curso de Liderazgo ha sentado precedentes mediante el establecimiento de estilos de enseñanza innovadoras. Dentro de esta modalidad, el estudiantado comparte la responsabilidad del éxito del curso con el profesorado.

También, debemos señalar que los estudiantes, como líderes gerenciales en su experiencia educativa durante el curso de liderazgo, han evidenciado que la gestión del talento humano es uno de los procesos más importantes hacia la consideración de la ventaja competitiva sostenible en una organización. Estudiosos como Lawler III (2008), indican que los gerentes también necesitan mostrarles a los empleados como ir de la teoría a la práctica.

Por otra parte, coincidimos con Lawler III, en que los gerenciales actuales que provienen de una escuela de negocios, "no necesariamente 
implica que poseen liderazgo, conocimiento y destrezas gerenciales”. También concluimos, que el uso del aprendizaje activo y cooperativo dentro y fuera del aula de liderazgo maximizó el aprendizaje estudiantil al crear un ambiente de mayor interacción fomentado la motivación, asistencia y la participación del estudiantado. Al mismo tiempo, la variedad en actividades de aprendizaje permitió el éxito en el cumplimiento del temario del curso. Implícitamente, el curso obtuvo una cobertura adicional de temas que mediante el proceso de enseñanza y aprendizaje que utilizando el método tradicional no se hubiera logrado.

Reconocemos que las instituciones de educación superior, se enfrentan constantemente a la siguiente interrogante: ¿para qué educamos?, ¿cómo los diseños curriculares contribuyen a la formación de futuros profesionales?, ¿en qué medida el compartir las experiencias pedagógicas añade valor a la obtención del conocimiento? Esta son algunas de las interrogantes que inciden en una educación superior en todos los campos de estudios como la Administración de Empresas y áreas relacionadas.

Recomendamos que se continúe investigando y fomentado el compartir las experiencias pedagógicas en el campo de la Administración de Empresas desde la perspectiva de la andragogía. Asimismo, recomendamos que el proceso de enseñanza y aprendizaje referente al enfoque de aprendizaje activo y cooperativo se promueva en otras disciplinas como una alternativa hacia una cultura de aprendizaje eficaz y específicamente el campo de la Administración de Empresas. 


\section{RefERENCIAS}

Argyris, C. (1991). Teaching smart people how to learn. Harvard Business Review, 69(3), 99-109.

Braxon, J. M., Milem, J. F. \& Sullivan, A. S. (2000). The influence of active learning on the college student departure process: Toward a revision of Tinto's Theory. The Journal of Higher Education, 71(5), 569-590.

Bonwell, C. C. \& Eison, J. A. (1991). Active learning: Creating excitement in the classroom. ERIC Clearinghouse on Higher Education. Washington, D.C.: George Washington University.

Caccavo, Jr., F. (2001). Teaching introductory microbiology with active learning. The American Biology Teacher 63(3), 172-175.

Claudio Tirado, R. (2002). Visiones y perspectiva de la educación puertorriqueña. San Juan, P.R.: Editorial Impresora Oriental.

Crouch, C. H. \& Mazur, E. (2001). Peer instruction: Ten years of experience and results. American Journal of Physics, 69(9), 970-979.

Departamento de Trabajo de Puerto Rico. (2005). Estudio de Destrezas y Ocupaciones en Mayor Demanda. Parte I. Informe de Destrezas. Estado Libre Asociado de Puerto Rico.

Davis, B. G. (1993). Tools for teaching. San Francisco, C.A.: Jossey-Bass Publishers.

Handelsman, J., Ebert-May D., Beichmer R., Buns P., Chang, A., DeHaan, R., Gentile, J., Lauffer, S., Stewart, J., Tilghman, S. M. \& Wood, W.B. (2004). Scientific teaching. Science, 304(5670), 521-522.

Hickcox, L. K. (2002). Personalizing teaching through experiential learning. College Teaching, 50(4), 123-128.

Hodgkinson, M. \& Brown, G. (2003). Enhancing the quality of education: A case study and some emerging principles. Higher Education, 45, 337-52.

Hughes, R., Ginnett, R. \& Curphy, G. (2007). Liderazgo: cómo aprovechar las lecciones de la experiencia. 5ta Edición. México: McGraw-Hill.

Human, S. E., Clark, T. \& Baucus, M. (2005). Student online selfassessment: Structuring individual-level learning in a new venture creation course. Journal of Management Education, 29(1), 111-134. 
Human, S. E., Kilbourne, L. M., Clark, T. D., Shriberg, A. \& Cunningham, W. (1999). Using web-enhanced instruction in an interpersonal skills course. Journal of Management Education, 23(5), 584-606.

Keyser, M. W. (2000). Active learning and cooperative learning: Understanding the difference and using both styles effectively. $R e-$ search Strategies, 17(1), 35-44.

Knight, J. K. \& Wood, W. B. (2005). Teaching more by lecturing less. Cell Biology Education, 4(4), 298-310.

Lawler III, E. E. (2008). Talent-Making people your competitive advantage. San Francisco, C.A.: Joseey-Bass Publishers.

Lunsford, B. E. \& Herzog, M. J. R. (1997). Active learning in anatomy and physiology: Students reactions and outcomes in a nontraditional A\&P course. The American Biology Teacher, 59(2), 80-85.

McKeachie, W. J. (1999). Teaching tips: Strategies, research and theory for college and university teachers. Boston: Houghton Mifflin.

Mazur, E. (1997). Peer instruction: A user's manual. Upper Saddle River, N.J.: Prentice Hall.

Meier, D. (2000). The accelerated learning handbook - A creative guide to designing and delivering faster, more effective training programs. New York: McGraw-Hill.

Meyers, C., \& Jones, T. B. (1993). Promoting active learning: Strategies for the college classroom. San Francisco, C.A: Jossey-Bass.

Petress, K. (2008). What is meant by "active learning"? Education, $128(4), 566$.

Schein, E. H. (1985). Organizational culture and leadership: A dynamic view. San Francisco, C.A.: Jossey-Bass.

Senge, P. (1990). The fifth discipline: The art and practice of the learning organization. New York: Doubleday/Currency.

Silberman, M. (1998). Active training - A handbook of techniques, designs, case examples, and tips. 2Ed. SanFrancisco, CA.: Jossey-Bass.

Silberman, M. (1996). Active learning: 101 strategies to teach any subject. Boston: Allyn \& Bacon.

Sullivan, S. \& Glanz, J. (2000). Supervision that improves teaching: Strategies and techniques. Thousand Oaks, C.A.: Corwin Press, Inc.

Tesier, J. T. (2004). Use of peer teaching to promote learning in biology. Journal of College Science Teaching, 33(6), 16-19. 
Tesier, J. T. (2006). Writing assignments in a nonmajor introductory ecology class. Journal of College Science Teaching, 35(4), 25-29.

Tesier, J. T. (2007). Small-group peer teaching in an introductory biology classroom. Journal of College Science Teaching, 36(4), 64-69.

UNESCO. (1998). Declaración mundial sobre la educación superior en el siglo XXI: visión y acción. Conferencia mundial sobre la educación.

UNESCO. (2003). Mesa redonda magistrar sobre la educación de calidad.

Verdejo, A. \& Medina, M. (2008). Evaluación del aprendizaje estudiantil. Cuarta Edición Aumentada. San Juan, P.R.: Isla Negra Editores.

Yukl, G. (2006). Leadership in organizations. 6ta Edición. n.l.: Prentice Hall.

Zemke, R., Raines, C. \& Filicpack, B. (2000). Generations at work: Managing the clash of veterans, boomers, xers, and nexters in your workplace. New York: AMACOM. 